\title{
MULTI-SCALE EXPANSIONS IN THE THEORY OF SYSTEMS INTEGRABLE BY THE INVERSE SCATTERING TRANSFORM
}

\author{
V.E. ZAKHAROV and E.A. KUZNETSOV \\ Landau Institute of Theoretical Physics, USSR Academy of Sciences, Moscow, USSR \\ and Institute of Automation and Electrometry, USSR Academy of Sciences, Novosibirsk, USSR
}

\begin{abstract}
It is shown that using multi-scale expansions conventionally employed in the theory of nonlinear waves one can transform systems integrable by the IST method into other systems of this type.
\end{abstract}

\section{Introduction}

The majority of nonlinear differential equations integrable by the inverse scattering transform (IST) and having physical applications represents different models of interacting waves. In the wave theory for nonlinear media there is a familiar totality of methods, which are applied in such cases when there exists two or several considerably differing scales. This totality is known under the general name of the multi-scale expansion method. This method allows to obtain for such situations new equations which differ from the initial ones and are more adequate to the given problem (see, e.g. $[1,2])$.

So, if it is already known that the solution represents a set of one or more quasimonochromatic wave packets of a small amplitude, it is reasonable to turn to a set of equations for complex envelopes of these packets. A characteristic packet size and wavelength play a role of different scales for this problem. If, on the contrary, we are interested in extremely long-wave weak nonlinear oscillations, the problem can be simplified by expanding the wave dispersion law in the neighbourhood of the wave number $k$ equal to zero, then by passing to the moving coordinate system we can eliminate the terms linear with respect to a wave number - the $\mathrm{KdV}$ equation is usually derived in this way.
It is important to note that these versions of the multiscale expansion method are structurally rough - they are equally applied both to the integrable and nonintegrable systems. If the initial system is nonintegrable, the result can be both integrable and nonintegrable. But if we treat the integrable system properly, we again must get from it an integrable system. That is why we became interested in application of multi-scale expansions to the integrable systems. We hoped thus to obtain new integrable formerly unknown systems.

In reality we found few such systems. One of them is shown in the end of the present paper. However, application of multi-scale expansions to the integrable systems in most cases leads to one of the classical equations which at one time made famous the inverse scattering transform - the nonlinear Schrödinger equation (NSE), the Korteweg-deVries equation (KdV), the DaveyStewartson equation (DS), the system of $N$-waves. This emphasizes once more the universality of the enumerated systems. This fact explains publication of this paper in the present collection of works surveying almost twenty years of history of IST (beginning from the first papers by $\mathrm{M}$. Kruskal and coworkers). We should like to show that in the first stage of development of this method many opportunities have been missed. Thus, from the fact of the applicability of IST to the KdV equa- 
tions it is not difficult to predict the application of this method to solution of the NSE. This can be made easier basing on a matrix version of the $\mathrm{KdV}$ equation. Using the latter it is easy to determine the applicability of IST to the $\mathrm{N}$-wave problem and to a vector version of the NSE. The integrability of NSE is also derived from the integrability of many other one-dimensional equations (Boussinesq equation, sine-Gordon equation and so on). The integrability of the Davey-Stewartson equation and a two-dimensional $N$-wave problem is derived similarly from the applicability of IST to the Kadomtsev - Petviashvili equation (KP).

We should also note, that the method we use is related to the known "averaging method" by Whitham [1] developed also in the works of Flaschka, Forest and McLaughlin [3]. This method allows to describe solutions of integrable systems which are locally close to finite-zone solutions. However, our method is more elementary and we can advance further on; in particular we can realize a correspondence between integrable systems not only on the level of the equations but also on the level of their integrating procedures $(L-A$ pairs, Marchenko equations and so on).

\section{Derivation of the nonlinear Schrödinger equation from the scalar Korteweg-deVries equation}

Consider the scalar $\mathrm{KdV}$ equation

$u_{t}+6 u u_{x}+u_{x x x}=0$.

The solution of it can be found in the form

$u=\sum_{n=-\infty}^{\infty} u_{n} \mathrm{e}^{\mathrm{i} n \theta}, \quad u_{n}^{*}=u_{-n}, \quad \theta=k^{3} t+k x$.

Here $k>0$ is an arbitrary number. Equating to zero coefficients at every $\mathrm{e}^{\mathrm{i} n \theta}$ separately, we get an infinite set of equations for $u_{n}$,

$$
\begin{aligned}
& \left(\frac{\partial}{\partial t}+\mathrm{i} n k^{3}\right) u_{n}+\left(\frac{\partial}{\partial x}+\mathrm{i} n k\right)^{3} u_{n} \\
& \quad+3\left(\frac{\partial}{\partial x}+\mathrm{i} n k\right) \sum_{q=-\infty}^{\infty} u_{q} u_{n-q}=0 .
\end{aligned}
$$

It is sufficient to consider the case $n \geq 0$. Let us introduce a parameter $\varepsilon$ to these equations, assuming

$u_{n}=\varepsilon^{\alpha_{n}} v_{n}\left(x^{\prime}, t^{\prime}\right)$.

Here

$x^{\prime}=\varepsilon\left(x+3 k^{2} t\right), \quad t^{\prime}=-6 \varepsilon^{2} k t, \quad \alpha_{0}=2$,

$\alpha_{n}=\alpha_{-n}=n \quad(n \geq 1)$.

Substitute (2.4), (2.5) into (2.3). Expression (2.3) becomes an infinite series with respect to $\varepsilon$. Let $\varepsilon \rightarrow 0$ and vanish the terms at minimal (for every $n)$ power of parameter $\varepsilon$. The system (2.3) is significantly reduced as $\varepsilon \rightarrow 0$ converting into explicit expressions for corresponding $V_{n}$ (except for the case $n=1$ ). In particular, we have

$V_{0}=-\frac{2}{k^{2}}\left|V_{1}\right|^{2}, \quad V_{2}=\frac{1}{k^{2}} V_{1}^{2}$.

The equation for $V_{1}$ remains differential. It has the form

$-\frac{\partial V_{1}}{\partial t^{\prime}}+\frac{\mathrm{i}}{2} \frac{\partial^{2} V_{1}}{\partial x^{\prime 2}}+\mathrm{i}\left(V_{0} V_{1}+V_{2} V_{1}^{*}\right)=0$.

Let us introduce the function $\psi=V_{1} / k$. Now, taking into account (2.6), (2.5) we have

$i \psi_{t^{\prime}}+\frac{1}{2} \frac{\partial^{2} \psi}{\partial x^{\prime 2}}-|\psi|^{2} \psi=0$

Thus, we got the nonlinear Schrödinger equation from the $\mathrm{KdV}$ equation. This fact is quite natural because expression (1.2) at $\varepsilon \rightarrow 0$ represents a quasi-monochromatic weakly nonlinear wave packet whose complex envelope should be described by the nonlinear Schrödinger equation.

It is less clear that correspondence between $\mathrm{KdV}$ and NSE can be established on the level of the integrable linear systems.

The $\mathrm{KdV}$ equation (2.1) is a compatibility condition for the following overdetermined system:

$$
\begin{aligned}
L \phi=\left(\frac{\partial^{2}}{\partial x^{2}}+u\right) \phi & +\lambda^{2} \phi=0 \\
M \phi=\left(\frac{\partial}{\partial t}+A\right) \phi & =\left(\frac{\partial}{\partial t}+4 \frac{\partial^{3}}{\partial x^{3}}+6 u \frac{\partial}{\partial x}+3 u_{x}\right) \Phi \\
& =0 .
\end{aligned}
$$


As before, let us represent $u$ in the form (2.2), and for $\Phi$ let us assume

$\Phi=\sum_{n=-\infty}^{\infty} \psi_{n} \mathrm{e}^{\mathrm{i} n \theta / 2}$

Here the summing up is taken over the even $n$. Introduce the parameter $\varepsilon$ into (2.11)

$\psi_{n}=\varepsilon^{(|n|-1) / 2} \Phi_{n}\left(x^{\prime}, t^{\prime}\right)$

The choice of $\Phi$ in the form of (2.11), (2.12) is associated with the fact that for periodic potentials in the limit of $\varepsilon \rightarrow 0$ points of the spectrum for the operator $L, \lambda_{n}^{2}=\left(n+\frac{1}{2}\right)^{2} k^{2}$, define the position of the forbidden zones. For potentials in the form of a quasimonochromatic wave (2.2) the reflection coefficient in these points has sharp peaks corresponding to the strong resonance subbarrier reflection.

Let us consider eq. (2.9) near the first resonance $\lambda=k / 2$. Let

$\lambda=\frac{k}{2}+\varepsilon \mu$.

Substituting (2.1), (2.11) into (2.9) and (2.10) and equating to zero coefficients at all $\mathrm{e}^{\mathrm{i} n \theta / 2}$ in turn, we get two infinite sets of equations for $\Phi_{n}$ (for the reason of brevity we do not insert them here). Each system represents an infinite series in powers of the parameter $\varepsilon$. Consider the system arising from eq. (2.9) and put $\varepsilon \rightarrow 0$ in it.

All the equations except corresponding $n= \pm 1$ are converted into explicit expressions for $\Phi_{n}$. In particular, we have

$\Phi_{3}=\frac{V_{1} \Phi_{1}}{2 k^{2}}, \quad \Phi_{-3}=\frac{V_{1}^{*} \Phi_{-1}}{2 k^{2}}$.

At $n= \pm 1$ we find

$\mathrm{i} \frac{\partial \Phi_{1}}{\partial x^{\prime}}+\psi \Phi_{-1}=\mu \Phi_{1}$,

$-\mathrm{i} \frac{\partial \Phi_{-1}}{\partial x^{\prime}}+\psi^{*} \Phi_{1}=\mu \Phi_{-1}$.

In the system (2.15) we find out the $L$-operator for the nonlinear Schrödinger equation (2.8). Similarly, substituting (2.2), (2.11), (2.12) into (2.10), consider $n= \pm 1$ and let $\varepsilon \rightarrow 0$. The terms of zero and first order in $\varepsilon$ are cancelled. Calculating the terms of second order we should take into account terms in the form of $V_{1}^{*} \Phi_{3}$ and $V_{1} \Phi_{-3}$. Using formulae (2.4) after simple calculations we finally find

$$
\begin{aligned}
& {\left[\frac{\partial}{\partial t^{\prime}}-\mathrm{i}\left(\begin{array}{rr}
1 & 0 \\
0 & -1
\end{array}\right) \frac{\partial^{2}}{\partial x^{2}}-\left(\begin{array}{cc}
0 & \psi \\
\psi^{*} & 0
\end{array}\right) \frac{\partial}{\partial x}\right.} \\
& \left.-\frac{1}{2}\left(\begin{array}{cc}
\mathrm{i}\left(v_{0}+|\psi|^{2}\right) & \psi_{x} \\
\psi_{x}^{*} & -\mathrm{i}\left(v_{0}+|\psi|^{2}\right)
\end{array}\right)\right]\left(\begin{array}{c}
\Phi_{1} \\
\Phi_{-1}
\end{array}\right) \\
& \quad=0 .
\end{aligned}
$$

Systems (2.15) and (2.16) are compatible when the quantity $V_{0}=-2|\psi|^{2}$ is in complete correspondence with (2.6) and $\psi$ obeys NSE.

Let us observe a correspondence between the $\mathrm{KdV}$ equation and the NS equation on the level of equations of the inverse scattering problem. Consider the Marchenko equation

$K(x, z)+F(x+z)+\int_{x}^{\infty} K(x, s) F(s+z) \mathrm{d} s=0$.

The potential $u(x)=2 \mathrm{~d} K(x, x) / \mathrm{d} x$ in the form of a quasimonochromatic wave packet with a mean wave number $k$ corresponds to the following choice of $F(\xi)$ :

$F(\xi)=\varphi(\xi) \mathrm{e}^{\mathrm{i} k \xi / 2}+\varphi^{*}(\xi) \mathrm{e}^{-\mathrm{i} k \xi / 2}$.

Function $K(x, z)$ should be sought in the form

$$
\begin{aligned}
& K(x, z) \\
& \quad=K_{0}(x, z) \mathrm{e}^{\mathrm{i}(k / 2)(x-z)}+K_{0}^{*}(x, z) \mathrm{e}^{-\mathrm{i}(k / 2)(x-z)} \\
& \quad+K_{1}(x, z) \mathrm{e}^{\mathrm{i}(k / 2)(x+z)}+K_{1}^{*}(x, z) \mathrm{e}^{-\mathrm{i}(k / 2)(x+z)} .
\end{aligned}
$$

In the formulae (2.18), (2.19) the functions $\varphi, k_{0}, k_{1}$ are varying on both arguments slowly relative to exponents. Substituting (2.18) and (2.19) 
into (2.18) and rejecting integrals from highfrequency oscillating function we finally get the equations

$$
\begin{aligned}
& K_{1}(x, z)+\varphi(x+z) \\
& \quad+\int_{x}^{\infty} K_{0}(x, s) \varphi(s+z) \mathrm{d} s=0, \\
& K_{0}(x, z)+\int_{x}^{\infty} K_{1}(x, s) \varphi^{*}(s+z) \mathrm{d} s=0,
\end{aligned}
$$

which coincide with the Marchenko equations for the linear problem (2.15).

The given scheme of NSE derivation is applicable (sometimes with small modifications) if we take as an initial equation any other integrable system, e.g. the Boussinesq or sine-Gordon equation. However, the integrability of these systems was discovered later than the integrability of the non-linear Schrödinger equation.

\section{Derivation of the nonlinear Schrödinger equation from the matrix Korteweg-deVries equation}

It is easier to derive NSE from the matrix $\mathrm{KdV}$ equation

$u_{t}+3\left(u^{2}\right)_{x}+u_{x x x}=0$.

Here $u$ is the complex $N \times N$ matrix. Let $N=2$ and $u$ Hermitian, $u=\left[\begin{array}{cc}u_{1} & w \\ w^{*} & u_{2}\end{array}\right], u_{1,2}$ are real. Eq. (3.1) is equivalent to the system

$$
\begin{aligned}
& \frac{\partial u_{1}}{\partial t}+\frac{\partial^{3} u_{1}}{\partial x^{3}}+3 \frac{\partial}{\partial x}\left(u_{1}^{2}+|w|^{2}\right)=0 \\
& \frac{\partial u_{2}}{\partial t}+\frac{\partial^{3} u_{2}}{\partial x^{3}}+3 \frac{\partial}{\partial x}\left(u_{2}^{2}+|w|^{2}\right)=0 \\
& \frac{\partial w}{\partial t}+\frac{\partial^{3} w}{\partial x^{3}}+3 \frac{\partial}{\partial x}\left(u_{1}+u_{2}\right) w=0 .
\end{aligned}
$$

System (2.2)-(2.4) has an exact simple solution

$u_{1}=a_{1}, \quad u_{2}=a_{2}, \quad w=\mathrm{e}^{\mathrm{i}(k x+\omega t)}$,

$\omega=k^{3}-3 k\left(a_{1}+a_{2}\right)$,

$a_{1,2}$ are arbitrary constants.
Let us perform a separation of scales in the system (3.2)-(3.4) considering solutions locally close to (3.5). Introduce a parameter $\varepsilon$, assuming

$u_{1}=a_{1}+\varepsilon^{2} V_{1}\left(x^{\prime}, t^{\prime}\right), \quad u_{2}=a_{2}+\varepsilon^{2} V_{2}\left(x^{\prime}, t^{\prime}\right)$,

$w=\varepsilon \mathrm{e}^{\mathrm{i}(\omega t+k x)} \psi\left(x^{\prime}, t^{\prime}\right)$,

$x^{\prime}=\varepsilon x, \quad t^{\prime}=-3 k \varepsilon^{2} t$.

From (3.2), (3.3) as $\varepsilon \rightarrow 0$ we obtain

$V_{1}=-\frac{|\psi|^{2}}{2 a_{1}}, \quad V_{2}=-\frac{|\psi|^{2}}{2 a_{2}}$.

As $\varepsilon \rightarrow 0$ in eq. (3.4) we demand the terms of second order in $\varepsilon$ to vanish. This gives the following condition:

$a_{1}+a_{2}=k^{2}$.

If we turn into zero the coefficient at $\varepsilon^{3}$ in (3.4) we obtain

$i \psi_{t^{\prime}}+\psi_{x^{\prime} x^{\prime}}-\alpha|\psi|^{2} \psi=0$

$\alpha=\frac{k^{2}}{2 a_{1} a_{2}}=\frac{k^{2}}{2 a_{1}\left(k^{2}-a_{1}\right)}$.

So, we got the nonlinear Schrödinger equation again. It should be noted that the constant $\alpha$ which characterizes the interaction, can have any sign depending on the choice of $k^{2}$ and $a_{1}$.

From the scalar $\mathrm{KdV}$ equation we could only get the equation with $\alpha>0$ (NSE with repulsion).

Let us observe the transformation of the $L-A$ pair for the matrix $\mathrm{KdV}$ equation to a corresponding pair for NSE. Let us start from the system (2.9), (2.10) in which $u=\left(\begin{array}{ll}u_{1} & w \\ w^{*} & u_{2}\end{array}\right)$ is a matrix, and $\Phi=\left(\begin{array}{l}\Phi_{1} \\ \Phi_{2}\end{array}\right)$ is a two-component vector. $\Phi$ will be found in the form

$$
\begin{aligned}
& \Phi_{1}=\mathrm{e}^{\mathrm{i}(p+k / 2) x+\mathrm{i}(q+\omega / 2) t} \chi_{1}, \\
& \Phi_{2}=\mathrm{e}^{\mathrm{i}(p-k / 2) x+\mathrm{i}(q-\omega / 2) t} \chi_{2} .
\end{aligned}
$$


Here $p, q$ are the unknown constants, $\chi_{1,2}$ depend on slow variables $x^{\prime}, t^{\prime}$. Eqs. (2.9), (2.10) are considered in the $\lambda$-plane near the point $\lambda=\lambda_{0}$ which together with constants $p$ and $q$ are determined from the requirement of vanishing in (2.9), (2.10) the terms of zero order in $\varepsilon$. From (2.9) we have

$$
\begin{aligned}
& -\left(p+\frac{k}{2}\right)^{2}+\lambda_{0}^{2}+a_{1}=0 \\
& -\left(p-\frac{k}{2}\right)^{2}+\lambda_{0}^{2}+a_{2}=0
\end{aligned}
$$

from which

$$
\begin{gathered}
p=\frac{a_{1}-a_{2}}{2 k}=\frac{2 a_{1}-k^{2}}{2 k}, \\
\lambda_{0}^{2}=\frac{a_{1}\left(a_{1}-k^{2}\right)}{k^{2}}=-\frac{1}{2 \alpha} .
\end{gathered}
$$

Now, by setting $\lambda=\lambda_{0}-\mu \varepsilon / 2 \lambda_{0}$ and taking the limit as $\varepsilon \rightarrow 0$ in (2.9) we get a system

$$
\begin{aligned}
& 2 \mathrm{i}\left(p+\frac{k}{2}\right) \frac{\partial \chi_{1}}{\partial x}+\psi \chi_{2}=\mu \chi_{1} \\
& 2 \mathrm{i}\left(p-\frac{k}{2}\right) \frac{\partial \chi_{2}}{\partial x}+\psi^{*} \chi_{1}=\mu \chi_{2}
\end{aligned}
$$

which represents one of the possible versions of the $L$-operator for NSE (3.9) (compare with [4]).

Note that in the case of NSE with attraction $(\alpha<0)$ the point $\lambda_{0}$ lies on the real axis. Similarly, from the requirement of vanishing the terms of zero order in $\varepsilon$ we find $q=2 p^{3}$. Simultaneously in (2.10) the first order terms are cancelled. To combine the terms proportional to $\varepsilon$ one can get the second linear system integrating NSE (3.9). It is not necessary to write it out here.

\section{Other applications of the matrix $\mathrm{KdV}$ equation}

The matrix $\mathrm{KdV}$ equation (3.1) represents a suitable object for application of different multiscale expansions. Thus, from this equation one can get the known system of " $N$-waves" (see, e.g. [11]). Let in (3.1) $u=u_{i j}$ be a Hermitian matrix of order $N$. Let us separate a diagonal part in it,

$u_{i j}=u_{i} \delta_{i j}+w_{i j}, \quad w_{i i}=0$,

and introduce $n$ real numbers $a_{i}(i=1, \ldots, n)$. Further we introduce parameter $\varepsilon$, assuming

$$
\begin{aligned}
& u_{i}=a_{i}^{2}+\varepsilon^{2} V_{i}\left(x^{\prime}, t^{\prime}\right), \quad x^{\prime}=\varepsilon x, \quad t^{\prime}=3 \varepsilon t, \\
& w_{i j}=\varepsilon k \mathrm{e}^{\mathrm{i}\left(a_{i}-a_{j}\right) x-2 \mathrm{i}\left(a_{i}^{3}-a_{j}^{3}\right) t} \psi_{i j}\left(x^{\prime}, t^{\prime}\right),
\end{aligned}
$$

Substitute (4.1), (4.2) into (3.1) and take the limit as $\varepsilon \rightarrow 0$. At $i \neq j$ there occurs a closed set of equations for $\psi_{i j}=\psi_{j i}^{*}$;

$$
\frac{\partial}{\partial t} \psi_{i k}+2 a_{i} a_{k} \frac{\partial \psi_{i k}}{\partial x}+2 \mathrm{i}\left(a_{i}-a_{k}\right) \sum_{j=1}^{n} \psi_{i j} \psi_{j k}=0
$$

The system (4.3) is a special case of the hyperbolic nonlinear system for $N$-waves $\left(N=\frac{1}{2} n(n-1)\right)$. In order to obtain the $L-A$ pair for this system let us turn to the system (2.9), (2.10) in which $\Phi$ represents now a column of $n$ elements. Let us put for them

$\Phi_{i}=\mathrm{e}^{i\left(a_{i} x-2 a_{i}^{3} t\right)} \chi_{i}\left(x^{\prime}, t^{\prime}\right), \quad \lambda^{2}=\varepsilon \mu$.

Substituting (4.1), (4.2), (4.4) into (2.9), (2.10) and setting $\varepsilon \rightarrow 0$ we obtain

$2 \mathrm{i} a_{k} \frac{\partial \chi_{k}}{\partial x^{\prime}}+\sum_{j=1}^{n} \psi_{k j} \chi_{j}+\mu \chi_{k}=0$,

$\frac{\partial \chi_{k}}{\partial t^{\prime}}-2 a_{k}^{2} \frac{\partial \chi_{k}}{\partial x^{\prime}}+\sum_{j=1}^{n}\left(a_{k}-a_{j}\right) \psi_{k j} \chi_{j}=0$.

The system (4.3) is the compatibility condition for systems (4.5) and (4.6).

Now let us obtain a vector analogue of NSE from the matrix $\mathrm{KdV}$ equation (see [5]). Let us set 
in the matrix $u$ defined according to (4.1),

$u_{1}=a_{1}+\varepsilon^{2} V\left(x^{\prime}, t^{\prime}\right), \quad u_{i+1}=a_{2}+\varepsilon^{2} V_{i}\left(x^{\prime}, t^{\prime}\right)$,

$a_{1}+a_{2}=k^{2}, \quad w_{1, i+1}=\varepsilon \psi_{i}\left(x^{\prime}, t^{\prime}\right) \mathrm{e}^{\mathrm{i}\left(k x-2 k^{3} t\right)}$,

$w_{i+1, j+1}=\varepsilon^{2} \psi_{i j}\left(x^{\prime}, t^{\prime}\right), \quad i, j=1, \ldots, n-1$,

$x^{\prime}=\varepsilon x, \quad t^{\prime}=-3 k \varepsilon^{2} t$

Substituting (4.7) into (3.1) and taking the limit $\varepsilon \rightarrow 0$, we obtain

$\psi_{i j}=-\frac{\psi_{i}^{*} \psi_{j}}{2 a_{2}}, \quad V_{i}=-\frac{\left|\psi_{i}\right|^{2}}{2 a_{2}}$

$V=-\frac{1}{2 a_{1}} \sum_{i=1}^{n-1}\left|\psi_{i}\right|^{2}$.

For $\psi_{i}$ we now have a system of equations

$\mathrm{i} \frac{\partial \psi_{i}}{\partial t}+\frac{\partial^{2} \psi_{i}}{\partial x^{2}}-\alpha \sum_{k=1}^{n-1}\left|\psi_{k}\right|^{2} \psi_{i}=0$

where $\alpha=k^{2} / 2 a_{1} a_{2}$ as before. The $L-A$ pair for eq. (4.9) can be easily deduced from (2.9), (2.10) by analogy with the $L-A$ pair for the system of $N$-waves. Basing on the matrix $\mathrm{KdV}$ equation one can form even more exotic systems of equations. We present a simpler one. Let $n=2$,

$u_{1}=\varepsilon^{2} u\left(x^{\prime}, t^{\prime}\right), \quad x^{\prime}=\varepsilon x, \quad t^{\prime}=\varepsilon^{3} t$,

$u_{2}=k^{2}+\mathcal{O}\left(\varepsilon^{4}\right), \quad \psi_{12}=\varepsilon^{2} \mathrm{e}^{\mathrm{i} \theta} \psi\left(x^{\prime}, t^{\prime}\right)$,

$\theta=k x-2 k^{3} t-3 \mathrm{i} \varepsilon^{2} k \int^{t} \lambda(t) \mathrm{d} t$.

By substituting (4.10) into (3.1) we have as $\varepsilon \rightarrow 0$

$\frac{\partial u}{\partial t}+\frac{\partial^{3} u}{\partial x^{3}}+3 \frac{\partial}{\partial x}\left(u^{2}+|\psi|^{2}\right)=0$,

$-\lambda(t) \psi+\psi_{x x}+u \psi=0$

The system (4.11) is easily integrable; the discussion of this problem is outside the framework of the present paper.
5. From the nonlinear Schrödinger equation to the KdV equation

To give a complete picture we show how with the help of the multi-scale expansion the $\mathrm{KdV}$ equation can be derived from the NSE with repulsion (2.8). Let us separate the amplitude and the phase in this equation assuming

$\psi=\sqrt{N} \mathrm{e}^{\mathrm{i} \phi}$.

Then

$N_{t}+\frac{\partial}{\partial x}(N V)=0$,

$V_{t}+V V_{x}+N_{x}=\frac{1}{2} \frac{\partial}{\partial x} N^{-1 / 2} \frac{\partial^{2}}{\partial x^{2}} N^{1 / 2}$,

where $V=\phi_{x}$.

If $N=1+\delta n(\delta n \ll 1)$ the equations (5.2), (5.3) describe the propagation of small amplitude waves with the dispersion law $\omega_{k}=\sqrt{k^{2}+\frac{1}{4} k^{4}}$. For long waves $k^{2} \ll 1$ one can introduce the parameter $\varepsilon$, assuming

$\phi=-t+\sum_{k=1}^{\infty} \varepsilon^{2 k-1} \phi_{k}\left(x^{\prime}, t^{\prime}\right)$,

$N=1+\sum_{k=1}^{\infty} \varepsilon^{2 k} n_{k}\left(x^{\prime}, t^{\prime}\right)$.

Here

$V=\sum_{k=1}^{\infty} \varepsilon^{2 k} V_{k}\left(x^{\prime}, t^{\prime}\right)$

$x^{\prime}=\varepsilon(x-t), \quad t^{\prime}=\varepsilon^{3} t$

Furthermore it is expedient to consider a halfdifference and a half-sum of eqs. (5.2) and (5.3). The half-difference of these equations contains terms of the order $\varepsilon^{3}$. Equating to zero a coefficient at these terms we obtain

$V_{1}\left(x^{\prime}, t^{\prime}\right)=n_{1}\left(x^{\prime}, t^{\prime}\right)$

Taking it into account the half-sum gives the $\mathrm{KdV}$ 
equation

$n_{1 t^{\prime}}+\frac{3}{2} n_{1} n_{1 x^{\prime}}-\frac{1}{8} n_{1 x^{\prime} x^{\prime} x^{\prime}}=0$.

Transformation from the $L$-operator for NSE to the $L$-operator for the $\mathrm{KdV}$ equation represents a standard nonrelativistic expansion in the onedimensional Dirac operator.

\section{Multi-scale expansions in the Kadomtsev- Petviashvili equation}

The interesting object for the application of the multi-scale expansions is the KadomtsevPetviashvili (KP) equation

$\frac{\partial}{\partial x}\left(u_{t}+6 u u_{x}+u_{x x x}\right)=-3 \beta^{2} u_{y y}$.

This equation is a compatibility condition for the following linear system:

$\left(\beta \frac{\partial}{\partial y}+\frac{\partial^{2}}{\partial x^{2}}+u\right) \phi=0$

$\frac{\partial \phi}{\partial t}+\left(4 \frac{\partial^{3}}{\partial x^{3}}+6 u \frac{\partial}{\partial x}+3 u_{x}-3 \beta w\right) \phi=0$,

$w_{x}=u_{y}$.

It is more natural to repeat the procedure described in section 2 for the equation (6.1). Present $u$ in the form of (2.2) and assume

$u_{n}=\varepsilon^{\alpha_{n}} V_{n}\left(x^{\prime}, y^{\prime}, t^{\prime}\right), \quad V_{1}=k \psi$

Here

$x^{\prime}=\varepsilon\left(x+3 k^{2} t\right), \quad y^{\prime}=\varepsilon k y, \quad t^{\prime}=-3 \varepsilon^{2} k t$.

This procedure differs from the one described in section 2 only in one point. The equation for $v_{0}$ now takes the form

$\left(\frac{\partial^{2}}{\partial x^{2}}+\beta^{2} \frac{\partial^{2}}{\partial y^{2}}\right) V_{0}+2 \frac{\partial^{2}}{\partial x^{2}}|\psi|^{2}=0$ and as before we have $V_{2}=\psi^{2}$. With regard for this the equation for $\psi$ takes the form

$\mathrm{i} \psi_{t}+\frac{1}{2}\left(\psi_{x x}+\beta^{2} \psi_{y y}\right)+|\psi|^{2} \psi+V_{0} \psi=0$.

The system (6.4), (6.5) is known as DaveyStewartson equations. The $L-A$ pair for it is calculated from eqs. (6.2), (6.3) according to the scheme described in section 2 and has the form

$$
\begin{aligned}
& \left(\beta \frac{\partial}{\partial y}+\mathrm{i} \sigma_{3} \frac{\partial}{\partial x}+\hat{\psi}\right) \phi=0 \\
& {\left[\frac{\partial}{\partial t}-\mathrm{i} \sigma_{3} \frac{\partial^{2}}{\partial x^{2}}-\hat{\psi} \frac{\partial}{\partial x}-\frac{1}{2} \hat{\psi}_{x}\right.} \\
& \left.-\mathrm{i} \frac{\sigma_{3}}{2}\left(V_{0}+\beta \hat{\psi}_{y}+\hat{\psi}^{2}\right)+\frac{\beta}{2} w_{0 y}\right] \phi=0 .
\end{aligned}
$$

Here

$w_{0 x}=V_{0 y}, \quad \hat{\psi}=\left(\begin{array}{cc}0 & \psi \\ \psi^{*} & 0\end{array}\right), \quad \sigma_{3}=\left(\begin{array}{cc}1 & 0 \\ 0 & -1\end{array}\right)$

It is important to note the following. The onedimensional Schrödinger equation (3.9) is a universal model for the description of propagation of narrow spectral wave packets in one-dimensional nonlinear media with dispersion.

In two-dimensional media the universal model takes a more general form (see [6])

$\mathrm{i} \psi_{t}+L_{1} \psi+\alpha|\psi|^{2} \psi+u \psi=0$,

$L_{2} u=L_{3}|\psi|^{2}$.

Here $L_{i}=\Pi_{i}^{\alpha \beta} \partial^{2} / \partial x_{\alpha} \partial x_{\beta}$ are the second order differential operators, in particular, $\Pi_{1}^{\alpha \beta}$ $=\frac{1}{2} \partial^{2} \omega / \partial k_{\alpha} \partial k_{\beta}, \omega(k)$ is the dispersion law of waves.

The specific case of the system (6.7) is the Davey-Stewartson equation (6.4), (6.5) and besides, the two-dimensional Schrödinger equation (when the operators are mutually proportional).

The system (6.7) is, as a rule, nonintegrable. In particular, it is the two-dimensional NSE that is nonintegrable. All not numerous integrable versions of the system (6.7) are enumerated in paper 
[7] (see also [8, 9]) and the Davey-Stewartson equation is one of them. It is clear that as a result of application of multi-scale expansions to the two-dimensional integrable systems we can obtain only these special integrable systems.

Properties of the KP equation (6.1) depend sufficiently on the sign of the quantity $\beta^{2}$. At $\beta^{2}<0$ this equation is called the KP-1 equation, and at $\beta^{2}>0$ the KP-2 equation.

We show that from the KP-1 equation a system of two-dimensional equations for $N$-waves can be obtained. This system directly generalizes the system (4.3).

Let us define $n$ real numbers $a_{k}(k=1, \ldots, n)$ and introduce $N=\frac{1}{2} n(n-1)$ phases

$\theta_{i k}=\xi_{i}-\xi_{k}, \quad \xi_{i}=a_{i} x-a_{i}^{2} y+4 a_{i}^{3} t$

Note that

$\theta_{i k}=-\theta_{k i}, \quad \theta_{i k}=\theta_{i j}+\theta_{j k}$

The solution of equation (6.1) (at $\beta=i$ ) can be found in the form

$u=\sum_{i \neq k} \varepsilon \psi_{i k}\left(x^{\prime}, t^{\prime}, y^{\prime}\right) \mathrm{e}^{\theta_{i k}}+\mathcal{O}\left(\varepsilon^{2}\right)$

Here

$\psi_{i k}=\psi_{k i}^{*}, \quad x^{\prime}=\varepsilon x, \quad t^{\prime}=6 \varepsilon t, \quad y^{\prime}=\varepsilon y$.

Representation (6.10) gives $u$ in the form of $N$ quasimonochromatic packets. The relations (6.9) for them denote the decay conditions

$\omega_{i k}=\omega_{i j}+\omega_{j k}$,

$p_{i k}=p_{i j}+p_{j k}$,

$q_{i k}=q_{i j}+q_{j k}$,

where $\omega=p^{3}+3 p^{2} / q$ is the dispersion law for waves with small amplitude for the KP-1 equation, and $p, q$ are $x, y$-components of the wave vectors parametrized by the quantities $a_{k}[8]$.
It is easy to see that as $\varepsilon \rightarrow 0$ the set of equations for $\psi_{i k}$ closes and takes the form

$$
\begin{aligned}
& \frac{\partial \psi_{i k}}{\partial t}+2 a_{i} a_{k} \frac{\partial \psi_{i k}}{\partial x}+\left(a_{i}+a_{k}\right) \frac{\partial \psi_{i k}}{\partial y} \\
& \quad+\mathrm{i}\left(a_{i}-a_{k}\right) \sum_{j=1}^{n} \psi_{i j} \psi_{j k}=0 .
\end{aligned}
$$

The system (6.11) is a natural two-dimensional generalization of the system (4.3). The $L-A$ pair for this system can be easily extracted from the $L-A$ pair for the KP equation.

Let us consider the system (6.2), (6.3) at $\beta=i$ and present $\phi$ in the form

$\phi=\sum_{i=1}^{n} \chi_{i}\left(x^{\prime}, y^{\prime}, t^{\prime}\right) \mathrm{e}^{\mathrm{i} \xi_{i}}+\mathcal{O}(\varepsilon)$

Substituting (6.10), (6.12) into (6.2), (6.3) we get as $\varepsilon \rightarrow 0$ two systems of equations for $\chi_{i}$. One of them coincides with the equation (4.6), the other is of the form

$\mathrm{i}\left(\frac{\partial \chi_{k}}{\partial y}+2 a_{k} \frac{\partial \chi_{k}}{\partial x}\right)+\sum_{j=1}^{n} \psi_{k j} \chi_{j}=0$

that is, represents a two-dimensional generalization of the system (4.5).

Let us also consider possibilities arising from application of multi-scale expansions to a matrix analogy of the KP equation. If $u$ and $w$ are supposed matrices in the system (6.2), (6.3) for $\beta=i$, then the following equations arise

$u_{t}+3\left(u^{2}\right)_{x}+u_{x x x}=3 w_{y}-3 \mathrm{i}[u, w]$,
$u_{y}=w_{x}$.

Applying procedures, described in sections 3 and 4 , to the system (6.13), we can get from it the DS equation and its vector analogy and besides, a two-dimensional system of $N$-waves. Let us discuss in detail the derivation from (6.15) a two- 
dimensional analogue of the system (4.11). Let

$u=\left(\begin{array}{cc}u_{1} & \phi \\ \phi^{*} & u_{2}\end{array}\right), \quad w=\left(\begin{array}{cc}w_{1} & \xi \\ \xi^{*} & w_{2}\end{array}\right)$,

$u_{1}=\varepsilon^{2} u\left(x^{\prime}, y^{\prime}, t^{\prime}\right), \quad w_{1}=\varepsilon^{2} w\left(x^{\prime}, y^{\prime}, t^{\prime}\right)$,

$x^{\prime}=\varepsilon x, \quad y^{\prime}=\varepsilon^{2} y, \quad t^{\prime}=\varepsilon^{3} t$,

$\phi=\varepsilon^{2} \mathrm{e}^{\mathrm{i}\left(k x-2 k^{3} t\right)} \psi\left(x^{\prime}, y^{\prime}, t^{\prime}\right)$,

$\xi=\varepsilon^{4} \mathrm{e}^{\mathrm{i}\left(k x-2 k^{3} t\right)} \chi\left(x^{\prime}, y^{\prime}, t^{\prime}\right)$,

$u_{2}=k^{2}+\mathcal{O}\left(\varepsilon^{4}\right), \quad w_{2}=\mathcal{O}\left(\varepsilon^{5}\right)$.

Let us substitute (6.14) into (6.13) and take the limit as $\varepsilon \rightarrow 0$. As a result we obtain a system of equations

$\frac{\partial}{\partial x}\left(u_{t}+6 u u_{x}+u_{x x x}\right)-3 u_{y y}=-3 \frac{\partial^{2}}{\partial x^{2}}|\psi|^{2}$,

$\mathrm{i} \psi_{y}=\psi_{x x}+u \psi$.

The system (6.15) can be applied in the theory of nonlinear waves. It appeared earlier in the papers by V. Melnikov [10]. The system (4.11) arises from (6.15) when

$u_{y}=0, \quad \psi=\mathrm{e}^{-\mathrm{i} \lambda(t) y} \tilde{\psi}$.

\section{Conclusion}

Now let us raise the following question: do the abovementioned results possess any other value besides methodical? Undoubtedly, they emphasize universality of such fundamental integrable systems as $\mathrm{KdV}$, NSE, the $N$-wave problem, the Davey-Stewartson equation. We suppose that these results will be useful for further development of the theory of fundamental two-dimensional systems - KP and DS equations, the twodimensional $N$-wave problem-which from our point of view, is far from being completed. Using multi-scale expansions one can effectively observe correspondence between different theories of equations.

Attempts can be made to apply multi-scale expansions to each of the numerous known integrable systems, for example, to those which are integrated by means of linear problems rational over the spectral parameter. As a result we shall obtain with most probability one of the abovementioned equations. However, after all we can not eliminate the possibility of getting something absolutely new.

In conclusion we would like to mention that the concepts of the present paper have been suggested long ago and discussed for more than 10 years by our friends who from time to time took part in appropriate calculations. The present paper is " a dry remains" of these discussions and calculations.

\section{Acknowledgements}

We should like to thank A.V. Mikhailov, E.A. Benilov and especially S.V. Manakov for their helpful participation in discussions and calculations.

\section{References}

[1] G.B. Whitham, Linear and Nonlinear Waves (Wiley - Interscience, New York, 1974).

[2] V.E. Zakharov, Isv. Vuz. Radiofizika 15, (1974) 431.

[3] H. Flaschka, G. Forest and D.W. Mclaughlin, Com. Pure. Appl. Math. 33 (1979) 739.

[4] V.E. Zakharov and A.B. Shabat, Soviet Phys. JETP 34 (1972) 62

[5] S.V. Manakov, Sov. Phys. JETP 38 (1974) 248.

[6] V.E. Zakharov and E.A. Kuznetsov. Hamiltonian Formalism for Systems of Hydrodynamic Type, Sov. Sci. Rev., Sect. C 4 (1984) 167.

[7] E.I. Shulman, Teor. Mat. Fiz. 56 (1983) 131 (in Russian).

[8] V.E. Zakharov and E.I. Shulman, Physica 1D (1980) 791.

[9] V.E. Zakharov and E.I. Shulman, Physica 4D (1982) 270.

[10] V.K. Melnikov, Mat. Sbornik 121 (163) (1983) 469 (in Russian).

[11] V.E. Zakharov, S.V. Manakov, S.P. Novikov and L.P. Pitaevskii, Soliton Theory-Method of Inverse Scattering Problem (Nauka, Moscow, 1980). 